\title{
Metaanálisis del uso de semillas y aceites en la dieta de ovejas y cabras
}

\author{
Andrés Luis Martínez Marín ${ }^{(1)}$, Nieves Núñez Sánchez(1), Ana Isabel Garzón Sigler ${ }^{(1)}$, Francisco Peña Blanco(1), \\ Valeriano Domenech García ${ }^{(1)}$ y Fuensanta Hernández Ruipérez ${ }^{(2)}$
}

(1)Universidad de Córdoba, Departamento de Producción Animal, Ctra. Madrid-Cádiz, Km 396, 14071 Córdoba, España. E-mail: pa1martm@uco.es, pa2nusan@uco.es, pa1gasia@uco.es, pa1peblf@uco.es, pa1dogav@uco.es (2)Universidad de Murcia, Departamento de Producción Animal, Campus de Espinardo, 30071, Murcia, España. E-mail: nutri@um.es

Resumen - El objetivo de este trabajo fue utilizar el metaanálisis para estudiar los resultados productivos de ovejas y cabras de ordeño, alimentadas con aceites y semillas ricas en ácidos grasos insaturados. Se utilizaron 22 experimentos con ovejas (63 tratamientos) y 21 con cabras (76 tratamientos). El consumo medio de grasa extra fue de $83,6 \pm 33,6 \mathrm{~g}$ por día en ovejas y $73,4 \pm 38,7 \mathrm{~g}$ por día en cabras. En ovejas, la grasa extra no afectó al consumo de materia seca, pero aumentó la producción de grasa (de 91,4 a 98,8 g por día) y redujo el contenido proteico (de 5,41 a 5,24\%) de la leche. No hubo diferencias en el extracto quesero. En cabras, la inclusión de grasa en la dieta redujo el consumo de materia seca (de 2,13 a 2,08 kg por día) y aumentó el porcentaje de grasa láctea (de 4,11 a 4,43\%) y el extracto quesero (de 7,41 a 7,76\%). La respuesta productiva de ovejas y cabras al consumo de dietas ricas en ácidos grasos insaturados es diferente. Sin embargo, el valor comercial de la leche no se ve afectado, debido a la ausencia de efectos negativos sobre el extracto quesero en ambas especies.

Términos para indexación: ácidos grasos insaturados, grasa, leche, producción animal, proteína.

\section{Meta-analysis of the use of oilseeds and oils in ewe and goat diets}

\begin{abstract}
The objective of this work was to use meta-analysis to study the productive results of ewe and goat fed oil and seed, rich in unsatured fat acid. Twenty-two experiments with ewes (63 treatments) and 21 experiments with goats ( 76 treatments) were used. Average extra fat intake was $83.6 \pm 33.6 \mathrm{~g}$ per day in ewes, and $73.4 \pm 38.7 \mathrm{~g}$ per day in goats. In ewes, extra fat intake did not affect dry matter intake, but increased milk fat yield (from 91.4 to $98.8 \mathrm{~g}$ per day) and reduced milk protein content (from 5.41 to 5.24\%). There were no differences in the sum of fat and protein contents. In goats, extra fat included in the diet reduced dry matter intake (from 2.13 to $2.08 \mathrm{~kg}$ per day), and increased milk fat percentage (from 4.11 to $4.43 \%$ ) and the sum of fat and protein contents (from 7.41 to $7.76 \%$ ). The yield responses to the intake of diets enriched with unsaturated fatty acids differ between ewes and goats. However, milk commercial value is not affected because of the absence of negative effects on the sum of fat and protein contents in both species.
\end{abstract}

Index terms: unsaturated fatty acids, fat, milk, animal production, protein.

\section{Introducción}

Clásicamente, la síntesis del conocimiento científico sobre un tema en particular se ha circunscrito a la revisión cualitativa de los resultados publicados sobre un mismo tema. La principal limitación de este procedimiento es la subjetividad, ya que la opinión de los autores influye en la importancia concedida a los trabajos revisados y los criterios para la inclusión de estos mismos trabajos están pobremente definidos. Por el contrario, el metaanálisis es una herramienta estadística que permite la revisión objetiva de la información procedente de trabajos de investigación aunque presenten diferencias en su metodología
(Sauvant et al., 2008). El uso del metaanálisis ha permitido extraer valiosas conclusiones cuando se ha aplicado a experimentos de Nutrición Animal (Eugène et al., 2008; Rabiee et al., 2012).

Por una parte, en los últimos años, ha adquirido especial interés la modificación de las proporciones de los ácidos grasos de la leche mediante la inclusión de semillas y aceites en la dieta de las hembras rumiantes, por su efecto potencial sobre la salud de los consumidores (Shingfield et al., 2013). Por otra parte, la bibliografía indica que los efectos sobre la producción y composición de la leche, cuando se añaden fuentes de grasa similares a la dieta, no son iguales en vacas, ovejas y cabras (Martínez Marín et al., 2013b). La 
leche de ovejas y cabras se destina mayoritariamente a la elaboración de queso y otros derivados lácteos, en muchas áreas del mundo, y es bien conocida la influencia de su composición físicoquímica en el rendimiento quesero (Morand-Fehr et al., 2007). La grasa añadida a la dieta podría afectar al valor comercial de la leche de ovejas y cabras, si ello ocasiona una disminución de la suma de los contenidos en grasa y proteína (Pirisi et al., 2007). Por tanto, convendría esclarecer el efecto que la adición de semillas y aceites a la dieta de ovejas y cabras tiene sobre la producción de leche y su contenido de grasa y proteína, ya que un efecto negativo sobre los resultados productivos podría restar interés a los ganaderos para modificar el perfil de ácidos grasos de la leche, especialmente en tanto no exista un sistema de pago diferencial de la leche producida relativo al mismo efecto.

El objetivo de este trabajo fue utilizar el metaanálisis para investigar el efecto de la adición de semillas oleaginosas y aceites vegetales a la dieta de ovejas y cabras, sobre el consumo de materia seca y la producción y la composición de la leche.

\section{Materiales y Métodos}

Los trabajos de investigación referidos a la inclusión de fuentes vegetales de grasa, en la dieta de ovejas lecheras, se buscaron en bases de datos bibliográficas -ISI Web of Science, Scopus, Pubmed, Google Scholar-, utilizándose como palabras clave "grasas vegetales", "semillas", “aceites", “ovejas", “cabras", "leche", y "grasa" en los idiomas español, inglés, francés y portugués. Se seleccionaron 21 trabajos de investigación con ovejas y 18 con cabras, en los que un tratamiento control, con una dieta sin grasa añadida, se comparó con uno o más tratamientos en los que la dieta fue enriquecida con una única semilla o aceite vegetal, ricos en ácidos grasos insaturados. Estos trabajos se publicaron entre los años 1998 y 2013. En ellos, se utilizaron 14 razas y tres cruces, mayoritariamente de origen europeo. La variedad de razas fue mayor en el caso de los trabajos de ovejas (nueve razas y dos cruces) que en los de cabras (cinco razas y un cruce). La raza Assaf tuvo la mayor representación en los experimentos con ovejas, mientras que las razas Malagueña y Alpina fueron las más utilizadas en los experimentos con cabras. En todos los experimentos, los animales permanecieron estabulados y recibieron raciones con proporciones variables de concentrados y forrajes conservados (Cuadro 1).

Los trabajos con ovejas incluyeron 22 experimentos y 63 tratamientos, de los cuales 26 correspondieron a dietas sin grasa extra, cinco a dietas enriquecidas en ácido oleico, 17 a dietas enriquecidas en ácido linoleico, y 15 a dietas enriquecidas en ácido $\alpha$-linolénico. Se utilizaron aceites en 21 tratamientos

Cuadro 1. Referencias de los trabajos científicos cuyos experimentos se incluyeron en el metaanálisis.

\begin{tabular}{|c|c|c|c|c|}
\hline Trabajo & Especie & Referencia & $\begin{array}{l}\text { Número de } \\
\text { experimento }\end{array}$ & $\mathrm{TE}^{(1)}$ \\
\hline 1 & Oveja & Zervas et al. (1998) & 1 & 2 \\
\hline 2 & Cabra & Mir et al. (1999) & 1 & 4 \\
\hline 3 & Oveja & Pol et al. (2001) & 1 & 2 \\
\hline 4 & Oveja & Chiofalo et al. (2004) & 1 & 2 \\
\hline 5 & Cabra & Bernard et al. (2005) & 1 & 3 \\
\hline 6 & Cabra & Lana et al. (2005) & 1 & 3 \\
\hline 7 & Cabra & Schmidely et al. (2005) & 1 & 6 \\
\hline 8 & Cabra & Andrade \& Schmidely (2006b) & 1 & 4 \\
\hline 9 & Cabra & Andrade \& Schmidely (2006a) & 1 & 4 \\
\hline 10 & Cabra & Maia et al. (2006) & 1 & 3 \\
\hline 11 & Oveja & Mele et al. (2006) & 1 & 4 \\
\hline 12 & cabra & Nudda et al. (2006) & 1 & 3 \\
\hline 13 & Oveja & Zhang et al. (2006c) & 1 & 4 \\
\hline 14 & Oveja & Zhang et al. (2006b) & 1 & 3 \\
\hline 15 & Oveja & Zhang et al. (2006a) & 1 & 4 \\
\hline 16 & Cabra & Silva et al. (2007) & 1 & 3 \\
\hline 17 & Cabra & Bouattour et al. (2008) & 1 & 2 \\
\hline 18 & Oveja & Gómez-Cortés et al. (2008a) & 1 & 2 \\
\hline 19 & Oveja & Gómez-Cortés et al. (2008b) & 1 & 2 \\
\hline 20 & Oveja & Hervás et al. (2008) & 1 & 2 \\
\hline 21 & Cabra & Mele et al. (2008) & 1 & 4 \\
\hline 22 & Cabra & Bernard et al. (2009) & 2 & 3 \\
\hline 23 & Oveja & Castro et al. (2009) & 1 & 2 \\
\hline 24 & Oveja & Gómez-Cortés et al. (2009) & 1 & 3 \\
\hline 25 & Cabra & Li et al. (2009) & 1 & 2 \\
\hline 26 & Cabra & Ollier et al. (2009) & 1 & 4 \\
\hline 27 & Oveja & Cieslak et al. (2010) & 2 & 3 \\
\hline 28 & Oveja & Toral et al. (2010b) & 1 & 2 \\
\hline 29 & Oveja & Toral et al. (2010a) & 1 & 2 \\
\hline 30 & Oveja & Caroprese et al. (2011) & 1 & 4 \\
\hline 31 & Oveja & Gallardo et al. (2011) & 1 & 2 \\
\hline 32 & Oveja & Gómez-Cortés et al. (2011a) & 1 & 6 \\
\hline 33 & Oveja & Gómez-Cortés et al. (2011b) & 1 & 4 \\
\hline 34 & Cabra & Martínez Marín et al. (2011a) & 1 & 4 \\
\hline 35 & Cabra & Titi et al. (2011) & 1 & 5 \\
\hline 36 & Cabra & Martínez Marín et al. (2012) & 3 & 4 \\
\hline 37 & Oveja & Mughetti et al. (2012) & 1 & 3 \\
\hline 38 & Cabra & Martínez Marín et al. (2013a) & 1 & 4 \\
\hline 39 & Oveja & Prieto et al. (2013) & 1 & 2 \\
\hline
\end{tabular}

${ }^{(1)} \mathrm{TE}$, tratamiento por experimento. 
(de los que tres aportaron principalmente ácido oleico, 15 aportaron ácido linoleico, y tres aportaron ácido $\alpha$-linolénico) y semillas enteras o procesadas en 16 tratamientos (de los que dos aportaron principalmente ácido oleico, dos aportaron ácido linoleico, y 12 aportaron ácido $\alpha$-linolénico). Los trabajos con cabras incluyeron 21 experimentos y un total de 76 tratamientos, de los que 26 correspondieron a dietas sin grasa añadida, 16 a dietas enriquecidas en ácido oleico, 24 a dietas enriquecidas en ácido linoleico, y 10 a dietas enriquecidas en ácido $\alpha$-linolénico. Se utilizaron aceites en 37 tratamientos (de los que 11 aportaron principalmente ácido oleico, 19 aportaron ácido linoleico, y siete aportaron ácido $\alpha$-linolénico) y semillas enteras o procesadas en 13 tratamientos (de los que cinco aportaron principalmente ácido oleico, cinco aportaron ácido linoleico, y tres aportaron ácido $\alpha$-linolénico). El perfil de ácidos grasos de la grasa extra fue similar en los experimentos realizados con ovejas y cabras (Cuadro 2). En el metaanálisis, los tratamientos sin grasa extra se agruparon para formar el tratamiento control (Nolip), y los tratamientos con grasa extra se agruparon para formar el tratamiento experimental (LIP).

Los análisis estadísticos se hicieron con el programa SAS 9.1 (SAS Institute Inc., Cary, NC, EUA). En todos los análisis, se utilizó el procedimiento Mixed de acuerdo con los modelos propuestos por Sauvant et al. (2008). Los datos se ponderaron con la raíz cuadrada del número de animales usados en cada tratamiento, mediante la declaración Weight. El nivel de significación se estableció a 5\% de probabilidad.

Cuadro 2. Contenido en ácidos grasos mayoritarios (media \pm desviación típica), expresado en porcentaje de los ácidos grasos totales, de las fuentes de grasa utilizadas en los experimentos incluidos en el metaanálisis.

\begin{tabular}{lcccc}
\hline Ácidos grasos & Especie & \multicolumn{3}{c}{ Fuente de grasa } \\
\cline { 3 - 5 } & & $\begin{array}{c}\text { Ácido } \\
\text { oleico }\end{array}$ & $\begin{array}{c}\text { Ácido } \\
\text { linoleico }\end{array}$ & $\begin{array}{c}\text { Ácido } \\
\alpha \text {-linolénico }\end{array}$ \\
\hline Ácido oleico & Oveja & $69,5 \pm 14,6$ & $27,1 \pm 8,8$ & $20,4 \pm 0,7$ \\
(C18:1 cis-9) & Cabra & $67,3 \pm 14,1$ & $23,7 \pm 3,3$ & $19,4 \pm 2,4$ \\
Ácido linoleico & Oveja & $14,8 \pm 7,6$ & $54,5 \pm 5,3$ & $15,2 \pm 2,2$ \\
(C18:2 cis-9,cis-12) & Cabra & $16,4 \pm 8,8$ & $57,2 \pm 4,7$ & $16,2 \pm 0,7$ \\
Ácido $\alpha$-linolénico & Oveja & $6,6 \pm 4,0$ & $1,7 \pm 2,9$ & $52,5 \pm 1,5$ \\
(C18:3 n-3) & Cabra & $5,0 \pm 4,3$ & $4,0 \pm 3,7$ & $52,0 \pm 3,2$ \\
\hline
\end{tabular}

\section{Resultados y Discusión}

Respecto al metadiseño, cabe destacar que hubo más datos procedentes de tratamientos con aceites que con semillas, tanto en los trabajos con ovejas (57 vs $43 \%$, respectivamente) como, sobre todo, en los realizados con cabras ( 74 vs $26 \%$, respectivamente). En los experimentos realizados con ovejas, hubo pocos tratamientos con fuentes de grasa ricas en ácido oleico (14\%), en comparación con aquellos que utilizaron fuentes de grasa ricas en linoleico y $\alpha$-linolénico (46 y $40 \%$, respectivamente). En cabras, el menor número de tratamientos correspondió al ácido $\alpha$-linolénico (20\%) frente a los que utilizaron ácido oleico y linoleico (32 y $48 \%$, respectivamente). Debido al insuficiente número de datos para el análisis estadístico, dichos posibles factores de interferencia no pudieron investigarse en el metaanálisis.

El peso vivo (PV) fue $65,2 \pm 16,6 \mathrm{~kg}$ y $57,1 \pm 8,2 \mathrm{~kg}$ en ovejas y cabras, respectivamente. En ovejas, el consumo medio de grasa en el tratamiento experimental (LIP) fue de $83,6 \pm 33,6 \mathrm{~g}$ por día y estuvo comprendido entre 28,8 y 165,2 g por día. En cabras, el consumo medio de grasa añadida fue de $73,4 \pm 38,7 \mathrm{~g}$ por día y estuvo comprendido entre 15,0 y 207,0 g por día. A partir de lo anterior, se puede calcular que las dietas del tratamiento LIP aportaron más ácidos grasos preformados $\left(1,15 \mathrm{y} 1,16 \mathrm{~g} \mathrm{~kg}^{-1}\right.$ PV por día en ovejas y cabras) y más energía neta de lactación (1,97 y 1,67 MJ por día), tomándose como referencia el informe del National Research Council (EUA) sobre las necesidades nutricionales de ganado lechero (Nutrient..., 2001), que las del tratamiento control (Nolip). La interferencia de la composición de la dieta en los resultados obtenidos, si existió, debió ser mínima, ya que no hubo diferencias en el contenido de forraje, proteína bruta y fibra neutrodetergente entre los tratamientos en ninguna de las especies (Cuadro 3 ).

No hubo diferencias en el consumo de materia de seca (MS) entre tratamientos en ovejas, pero el consumo de MS fue menor en el tratamiento LIP en cabras (Cuadro 4). La reducción en el consumo de MS en cabras pudo deberse a que la grasa extra de la dieta ralentizó el vaciado ruminal, bien por disminución de la digestión ruminal de las paredes vegetales o por efectos metabólicos (Chilliard et al., 1993). Martínez Marín et al. (2011b) señalaron que la adición de grasa a la dieta de los rumiantes generalmente reduce la digestión de las paredes vegetales, cuando el nivel de 
inclusión es superior a 4\% MS. En el presente trabajo, 14 tratamientos contenían más de $4 \%$ de grasa añadida, habiéndose encontrado diferencias significativas del consumo de MS en seis de ellos respecto a los correspondientes controles (Bernard et al., 2005; Silva et al., 2007; Bernard et al., 2009). Por lo tanto, la disminución de la digestión ruminal de las paredes vegetales pudo causar el menor consumo de MS observado. Por el contrario, los efectos metabólicos fueron poco probables, porque los trabajos de Teh et al. (1994) y Brown-Crowder et al. (2001) sugieren que es necesario un consumo de grasa superior a $2,5 \mathrm{~g} \mathrm{~kg}^{-1}$ PV para que dichos efectos sean aparentes, y ese valor solamente se alcanzó en un tratamiento (Ollier et al., 2009).

En ovejas, la grasa extra pareció utilizarse simultáneamente como fuente de energía para la producción de leche y como fuente de ácidos grasos preformados para la síntesis de grasa láctea, causando aumentos numéricos no significativos $(P=0,08$ y $\mathrm{P}=0,31$ ) de la leche producida y del contenido graso en el tratamiento LIP (Cuadro 4). Estos aumentos simultáneos explicarían la mayor producción de grasa láctea observada en el tratamiento LIP. En cabras, la energía aportada por la grasa extra pudo compensar la menor disponibilidad de energía debida a la reducción del consumo de MS, de forma que la producción lechera fue similar entre tratamientos. La ausencia de variación en la producción lechera y la mayor disponibilidad de ácidos grasos preformados de cadena larga, en el tratamiento LIP, pudieron contribuir al aumento del porcentaje de grasa láctea, aunque no hubo efectos claros sobre su producción $(\mathrm{P}=0,11)$. Estos resultados están en coincidencia con lo señalado por Schmidely \& Sauvant (2001), Chilliard et al. (2003) y Sampelayo et al. (2007). Además, los resultados observados pudieron estar influidos por el momento de la lactación, en que se realizaron las recogidas de muestras en cada uno de los períodos de los experimentos incluidos en el metaanálisis $(97 \pm 61$ y $124 \pm 48$ días tras el parto en ovejas y cabras, respectivamente). Es bien conocido que el reparto de nutrientes en el organismo varía con el estado de la lactación, y la síntesis de grasa en el tejido adiposo predomina sobre la utilización de los ácidos grasos de cadena larga en la ubre, tras el pico de lactación (Chilliard et al., 2003). Sin embargo, los datos disponibles no permitieron considerar este posible factor de interferencia en el metaanálisis.

El contenido proteico de la leche fue menor en las ovejas del tratamiento LIP (Cuadro 4). Estos resultados coinciden con lo observado en respuesta a la inclusión de jabones cálcicos de ácidos grasos en

Cuadro 3. Composición de las dietas, en porcentaje de la materia seca, en los tratamientos de los experimentos incluidos en el metaanálisis.

\begin{tabular}{|c|c|c|c|c|c|c|c|c|}
\hline \multirow[t]{2}{*}{ Item } & \multicolumn{2}{|c|}{ Ovejas - tratamiento } & \multirow[t]{2}{*}{$\mathrm{EEM}^{(3)}$} & \multirow[t]{2}{*}{$\mathrm{p}$} & \multicolumn{2}{|c|}{ Cabras - tratamiento } & \multirow[t]{2}{*}{ EEM } & \multirow[t]{2}{*}{$\mathrm{p}$} \\
\hline & Nolip $^{(1)}$ & $\operatorname{LIP}^{(2)}$ & & & Nolip & LIP & & \\
\hline Forraje & 44,6 & 44,6 & 2,65 & 0,99 & 42,9 & 44,0 & 1,36 & 0,55 \\
\hline Proteína bruta & 16,7 & 16,5 & 2,60 & 0,05 & 15,9 & 16,2 & 0,21 & 0,30 \\
\hline Fibra neutrodetergente & 34,2 & 34,2 & 1,31 & 0,97 & 35,6 & 35,3 & 0,78 & 0,57 \\
\hline
\end{tabular}

${ }^{(1)}$ Nolip, dietas sin grasa añadida. ${ }^{(2)}$ LIP, dietas enriquecidas con grasa rica en ácido oleico, ácido linoleico o ácido $\alpha$-linolénico. ${ }^{(3)}$ EEM, error estándar de la media. La grasa extra incluida en el tratamiento LIP fue 3,24 $\pm 0,273 \%$ para ovejas y 3,36 $\pm 0,201 \%$ para cabras.

Cuadro 4. Efectos de la adición de grasa extra a la dieta de ovejas y cabras sobre la producción y la composición de la leche.

\begin{tabular}{|c|c|c|c|c|c|c|c|c|}
\hline \multirow[t]{2}{*}{ Item } & \multicolumn{2}{|c|}{ Ovejas - tratamiento } & \multirow[t]{2}{*}{$\mathrm{EEM}^{(3)}$} & \multirow[t]{2}{*}{$\mathrm{p}$} & \multicolumn{2}{|c|}{ Cabras - tratamiento } & \multirow[t]{2}{*}{ EEM } & \multirow[t]{2}{*}{$\mathrm{p}$} \\
\hline & Nolip$^{(1)}$ & $\operatorname{LIP}^{(2)}$ & & & NOLIP & LIP & & \\
\hline Consumo de materia seca (kg por día) & 2,56 & 2,58 & 0,08 & 0,70 & 2,13 & 2,08 & 0,07 & 0,04 \\
\hline Producción de leche (g por día) & 1516 & 1571 & 95,4 & 0,08 & 2331 & 2305 & 109,0 & 0,68 \\
\hline Grasa $(\%)$ & 6,39 & 6,50 & 0,138 & 0,31 & 4,11 & 4,43 & 0,124 & $<0,001$ \\
\hline Grasa (g por día) & 91,4 & 98,8 & 5,16 & $<0,001$ & 89,6 & 95,2 & 3,57 & 0,11 \\
\hline Proteína (\%) & 5,41 & 5,24 & 0,086 & $<0,01$ & 3,28 & 3,31 & 0,046 & 0,43 \\
\hline Proteína (g por día) & 77,8 & 79,5 & 4,27 & 0,29 & 74,8 & 73,3 & 3,30 & 0,44 \\
\hline Extracto quesero $^{(4)}(\%)$ & 11,80 & 11,74 & 0,211 & 0,69 & 7,41 & 7,76 & 0,157 & $<0,01$ \\
\hline
\end{tabular}

${ }^{(1)}$ Nolip, dietas sin grasa añadida. ${ }^{(2)}$ LIP, dietas enriquecidas con grasa rica en ácido oleico, ácido linoleico o ácido $\alpha$-linolénico ${ }^{(3)}$ EEM, error estándar de la media. ${ }^{(4)}$ Suma de los porcentajes de grasa y proteína. 
dietas para ovejas (Gargouri et al., 2006). Las causas de la reducción del contenido proteico de la leche observada en ovejas, cuando se incluyen fuentes de grasa en la dieta, no han sido aclaradas. Las hipótesis elaboradas para explicar el mismo efecto en vacas se centran en una posible escasez de aminoácidos en la glándula mamaria, que impediría sostener la síntesis de proteína al mismo ritmo que el aumento de la producción de leche, tras la suplementación con grasa (Wu \& Huber, 1994). La insuficiente disponibilidad de aminoácidos para la síntesis proteica mamaria podría deberse a una combinación de cambios digestivos (Coppock \& Wilks, 1991), metabólicos (Lobley, 1992) y hormonales (Casper \& Schingoethe, 1989; Palmquist \& Moser, 1981) derivados del consumo de grasa extra.

A diferencia de lo observado en las ovejas, el tratamiento LIP no tuvo efecto sobre el porcentaje de proteína láctea en cabras. Actualmente, se desconocen los motivos por los que la adición de grasa extra a las dietas de cabras no afectó negativamente al contenido de proteína láctea, en contraposición a lo observado en ovejas. Se ha especulado que podrían existir diferencias interespecíficas en los efectos que la adición de grasa a la dieta tiene sobre el equilibrio y la disponibilidad de sustratos para el metabolismo mamario (Chilliard et al., 2003; Sampelayo et al., 2007).

Respecto al extracto quesero (suma de los contenidos de grasa y proteína), la ausencia de diferencias entre tratamientos en ovejas se debió probablemente a que el aumento numérico del porcentaje de grasa, en el tratamiento LIP, fue suficiente para compensar la reducción del porcentaje de proteína en la leche de dicho tratamiento (Cuadro 4). En cabras, el aumento del porcentaje de grasa láctea y la ausencia de efectos negativos sobre el contenido proteico ocasionó un aumento del extracto quesero de la leche en el tratamiento LIP. Estos resultados indican que la inclusión de semillas oleaginosas o sus aceites, en la dieta de las ovejas y cabras, no repercutiría negativamente sobre el valor comercial de la leche, en un sistema de pago referenciado al extracto quesero (Pirisi et al., 2007). De hecho, no se han observado efectos negativos sobre la producción de queso tras la inclusión de semillas oleaginosas en la dieta de ovejas (Zhang et al., 2006b). Este resultado es relevante, porque numerosos estudios indican que la adición de semillas y aceites a la dieta de ovejas y cabras es una herramienta valiosa para modificar los contenidos de ácidos grasos de la leche, en un sentido favorable para la salud humana (Shingfield et al., 2013; Nudda et al., 2014), y que dichos cambios se mantienen en el queso obtenido (Zhang et al., 2006b; Bodas et al., 2010; Mele et al., 2011).

\section{Conclusiones}

1. La inclusión de semillas y aceites, ricos en ácidos grasos insaturados, en la dieta de ovejas, aumenta la producción de leche y reduce el contenido de proteína láctea.

2. La inclusión de semillas y aceites, ricos en ácidos grasos insaturados, en dietas de cabras, disminuye el consumo de materia seca y aumenta el porcentaje de grasa de la leche.

3. Dado que el extracto quesero no se afecta negativamente ni en ovejas ni en cabras, las semillas $\mathrm{y}$ aceites pueden incluirse en su dieta para modificar el perfil de ácidos grasos de la grasa láctea.

\section{Agradecimientos}

A los profesores del Departamento de Producción Animal, de la Universidad de Murcia (España), por su acogida y apoyo durante la formación posdoctoral del primer autor de este trabajo. Al profesor Gustavo Gómez Castro, del Departamento de Producción Animal, de la Universidad de Córdoba, por la lectura crítica y la corrección del manuscrito original.

\section{Referencias}

ANDRADE, P.V.D.; SCHMIDELY, P.H. Effect of duodenal infusion of trans 10 , cis12-CLA on milk performance and milk fatty acid profile in dairy goats fed high or low concentrate diet in combination with rolled canola seed. Reproduction Nutrition Development, v.46, p.31-48, 2006a. DOI: 10.1051/rnd:2005062.

ANDRADE, P.V.D.; SCHMIDELY, P.H. Influence of percentage of concentrate in combination with rolled canola seeds on performance, rumen fermentation and milk fatty acid composition in dairy goats. Livestock Science, v.104, p.77-90, 2006b. DOI: 10.1016/j.livsci.2006.03.010.

BERNARD, L.; ROUEL, J.; LEROUX, C.; FERLAY, A.; FAULCONNIER, Y.; LEGRAND, P.; CHILLIARD, Y. Mammary lipid metabolism and milk fatty acid secretion in alpine goats fed vegetable lipids. Journal of Dairy Science, v.88, p.1478-1489, 2005. DOI: 10.3168/jds.S0022-0302(05)72816-2.

BERNARD, L.; SHINGFIELD, K.J.; ROUEL, J.; FERLAY, A.; CHILLIARD, Y. Effect of plant oils in the diet on performance and milk fatty acid composition in goats fed diets based on grass hay 
or maize silage. British Journal of Nutrition, v.101, p.213-224, 2009. DOI: $10.1017 / \mathrm{S} 0007114508006533$.

BODAS, R.; MANSO, T.; MANTECÓN, A.R.; JUÁREZ, M.; DE LA FUENTE, M.A.; GÓMEZ-CORTÉS, P. Comparison of the fatty acid profiles in cheeses from ewes fed diets supplemented with different plant oils. Journal of Agricultural and Food Chemistry, v.58, p.10493-10502, 2010. DOI: 10.1021/jf101760u.

BOUATTOUR, M.A.; CASALS, R.; ALBANELL, E.; SUCH, X.; CAJA, G. Feeding soybean oil to dairy goats increases conjugated linoleic acid in milk. Journal of Dairy Science, v.91, p.23992407, 2008. DOI: 10.3168/jds.2007-0753.

BROWN-CROWDER, I.E.; HART, S.P.; CAMERON, M.; SAHLU, T.; GOETSCH, A.L. Effects of dietary tallow level on performance of Alpine does in early lactation. Small Ruminant Research, v.39, p.233-241, 2001. DOI: 10.1016/S0921-4488(00)00197-8.

CAROPRESE, M.; ALBENZIO, M.; BRUNO, A.; FEDELE, V.; SANTILLO, A.; SEVI, A. Effect of solar radiation and flaxseed supplementation on milk production and fatty acid profile of lactating ewes under high ambient temperature. Journal of Dairy Science, v.94, p.3856-3867, 2011. DOI: 10.3168/jds.2010-4067.

CASPER, D.P.; SCHINGOETHE, D.J. Model to describe and alleviate milk protein depression in early lactation dairy cows fed a high fat diet. Journal of Dairy Science, v.72, p.3327-3335, 1989. DOI: 10.3168/jds.S0022-0302(89)79494-7.

CASTRO, T.; MANSO, T.; JIMENO, V.; DEL ALAMO, M.; MANTECÓN, A.R. Effects of dietary sources of vegetable fats on performance of dairy ewes and conjugated linoleic acid (CLA) in milk. Small Ruminant Research, v.84, p.47-53, 2009. DOI: 10.1016/j.smallrumres.2009.05.005.

CHILLIARD, Y.; DOREAU, M.; GAGLIOSTRO, G.; ELMEDDAH, Y. Addition de lipides protégés (encapsulés ou savons de calcium) à la ration de vaches laitières. Effets sur les performances et la composition du lait. INRA Productions Animales, v.6, p.139-150, 1993.

CHILliARD, Y.; FERLAY, A.; ROUEL, J.; LAMBERET, G. A review of nutritional and physiological factors affecting goat milk lipid synthesis and lipolysis. Journal of Dairy Science, v.86, p.1751-1770, 2003. DOI: 10.3168/jds.S0022-0302(03)73761-8.

CHIOFAlO, B.; LIOTTA, L.; ZUMBO, A.; CHIOFALO, V. Administration of olive cake for ewe feeding: effect on milk yield and composition. Small Ruminant Research, v.55, p.169-176, 2004. DOI: 10.1016/j.smallrumres.2003.12.011.

CIESLAK, A.; KOWALCZYK, J.; CZAUDERNA, M.; POTKANSKI, A.; SZUMACHER-STRABEL, M. Enhancing unsaturated fatty acids in ewe's milk by feeding rapeseed or linseed oil. Czech Journal of Animal Science, v.55, p.496-504, 2010.

COPPOCK, C.E.; WILKS, D.L. Supplemental fat in high-energy rations for lactating cows: effects on intake, digestion, milk yield, and composition. Journal of Animal Science, v.69, p.3826-3837, 1991.

EUGÈNE, M.; MASSÉ, D.; CHIQUETTE, J.; BENCHAAR, C. Meta-analysis on the effects of lipid supplementation on methane production in lactating dairy cows. Canadian Journal of Animal Science, v.88, p.331-337, 2008. DOI: 10.4141/CJAS07112.
GALLARDO, B.; MANCA, M.G.; GUERRA, C.; BODAS, R.; MANTECÓN, A.R.; MANSO, T. Empleo de aceite de linaza en la ración de ovejas churras: efecto sobre la producción de leche, el crecimiento y las caracteristicas de la canal de los lechazos. In: JORNADAS SOBRE PRODUCCIÓN ANIMAL, 14., 2011, Zaragoza. [Anales]. Zaragoza: Asociación Interprofesional para el Desarrollo Agrario, 2011. p.649-651.

GARGOURI, A.; CAJA, G.; CASALS, R.; MEZGHANI, I. Lactational evaluation of effects of calcium soap of fatty acids on dairy ewes. Small Ruminant Research, v.66, p.1-10, 2006. DOI: 10.1016/j.smallrumres.2006.03.004.

GÓMEZ-CORTÉS, P.; BACH, A.; LUNA, P.; JUÁREZ, M.; DE LA FUENTE, M.A. Effects of extruded linseed supplementation on n-3 fatty acids and conjugated linoleic acid in milk and cheese from ewes. Journal of Dairy Science, v.92, p.4122-4134, 2009. DOI: $10.3168 /$ jds.2008-1909.

GÓMEZ-CORTÉS, P.; DE LA FUENTE, M.A.; TORAL, P.G.; FRUTOS, P.; JUÁREZ, M.; HERVÁS, G. Effects of different forage:concentrate ratios in dairy ewe diets supplemented with sunflower oil on animal performance and milk fatty acid profile. Journal of Dairy Science, v.94, p.4578-4588, 2011a. DOI: 10.3168/jds.2010-3803.

GÓMEZ-CORTÉS, P.; FRUTOS, P.; MANTECÓN, A.R.; JUÁREZ, M.; DE LA FUENTE, M.A.; HERVÁS, G. Milk production, conjugated linoleic acid content, and in vitro ruminal fermentation in response to high levels of soybean oil in dairy ewe diet. Journal of Dairy Science, v.91, p.1560-1569, 2008a. DOI: 10.3168/jds.2007-0722.

GÓMEZ-CORTÉS, P.; FRUTOS, P.; MANTECÓN, A.R.; JUÁREZ, M.; DE LA FUENTE, M.A.; HERVÁS, G. Addition of olive oil to dairy ewe diets: effect on milk fatty acid profile and animal performance. Journal of Dairy Science, v.91, p.31193127, 2008b. DOI: 10.3168/jds.2007-0954.

GÓMEZ-CORTÉS, P.; TORAL, P.G.; FRUTOS, P.; JUÁREZ, M.; DE LAFUENTE, M.A.; HERVÁS, G. Effect of the supplementation of dairy sheep diet with incremental amounts of sunflower oil on animal performance and milk fatty acid profile. Food Chemistry, v.125, p.644-651, 2011b. DOI: 10.1016/j.foodchem.2010.09.053.

HERVÁS, G.; LUNA, P.; MANTECÓN, A.R.; CASTAÑARES, N.; DE LA FUENTE, M.A.; JUÁREZ, M.; FRUTOS, P. Effect of diet supplementation with sunflower oil on milk production, fatty acid profile and ruminal fermentation in lactating dairy ewes. Journal of Dairy Research, v.75, p.399-405, 2008. DOI: 10.1017/ S0022029908003506.

LANA, R. de P.; CAMARDELLI, M.M.L.; QUEIROZ, A.C. de; RODRIGUES, M.T.; EIFERT, E. da C.; MIRANDA, E.N.; ALMEIDA, I.C.C. Óleo de soja e própolis na alimentação de cabras leiteiras. Revista Brasileira de Zootecnia, v.34, p.650-658, 2005. DOI: 10.1590/S1516-35982005000200035.

LI, X.Z.; YAN, C.G.; LONG, R.J.; JIN, G.L.; KHUU, J.S.; JI, B.J.; CHOI, S.H.; LEE, H.G.; SONG, M.K. Conjugated linoleic acid in rumen fluid and milk fat, and methane emission of lactating goats fed a soybean oil-based diet supplemented with sodium bicarbonate and monensin. Asian-Australasian Journal of Animal Sciences, v.22, p.1521-1530, 2009. DOI: 10.5713/ajas.2009.90073. 
LOBLEY, G.E. Control of the metabolic fate of amino acids in ruminants: a review. Journal of Animal Science, v.70, p.3264-3275, 1992.

MAIA, F.J.; BRANCO, A.F.; MOURO, G.F.; CONEGLIAN, S.M.; SANTOS, G.T. dos; MINELLA, T.F.; GUIMARÃES, K.C. Inclusão de fontes de óleo na dieta de cabras em lactação: produção, composição e perfil dos ácidos graxos do leite. Revista Brasileira de Zootecnia, v.35, p. 1504-1513, 2006. DOI: 10.1590/ S1516-35982006000500033.

MARTÍNEZ MARÍN, A.L.; GÓMEZ-CORTÉS, P.; GÓMEZ CASTRO, A.G.; JUÁREZ, M.; PÉREZ ALBA, L.M.; PÉREZ HERNÁNDEZ, M.; DE LA FUENTE, M.A. Animal performance and milk fatty acid profile of dairy goats fed diets with different unsaturated plant oils. Journal of Dairy Science, v.94, p.53595368, 2011a. DOI: 10.3168/jds.2011-4569.

MARTÍNEZ MARÍN, A.L.; GÓMEZ-CORTÉS, P.; GÓMEZ CASTRO, A.G.; JUÁREZ, M.; PÉREZ ALBA, L.M.; PÉREZ HERNÁNDEZ, M.; DE LA FUENTE, M.A. Effects of feeding increasing dietary levels of high oleic or regular sunflower or linseed oil on fatty acid profile of goat milk. Journal of Dairy Science, v.95, p.1942-1955, 2012. DOI: 10.3168/jds.2011-4303.

MARTÍNEZ MARÍN, A.L.; GÓMEZ-CORTÉS, P.; PÉREZ ALBA, L.M.; JUÁREZ, M.; GÓMEZ CASTRO, A.G.; PÉREZ HERNÁNDEZ, M.; DE LA FUENTE, M.A. Adición de aceites vegetales a la dieta de cabras lecheras: efecto sobre el contenido de ácidos grasos de la grasa láctea. Archivos de medicina veterinaria, v.45, p.259-266, 2013a. DOI: 10.4067/S0301732 X2013000300005.

MARTÍNEZ MARÍN, A.L.; PÉREZ HERNÁNDEZ, M.; PÉREZ ALBA, L.M.; CARRIÓN PARDO, D.; GARZÓN SIGLER, A.I.; GÓMEZ CASTRO, G. Fat addition in the diet of dairy ruminants and its effects on productive parameters. Revista Colombiana de Ciencias Pecuarias, v.26, p.69-78, 2013 b.

MARTÍNEZ MARÍN, A.L.; PÉREZ HERNÁNDEZ, M.; PÉREZ ALBA, L.M.; GÓMEZ-CASTRO, G.; CARRIÓN-PARDO, D. Efecto de las fuentes de grasa sobre la digestión de la fibra en los rumiantes. REDVET. Revista Electrónica de Veterinaria, v.12, p.1-22, 2011b.

MELE, M.; BUCCIONI, A.; PETACCHI, F.; SERRA, A.; BANNI, S.; ANTONGIOVANNI, M.; SECCHIARI, P. Effect of forage/ concentrate ratio and soybean oil supplementation on milk yield, and composition from Sarda ewes. Animal Research, v.55, p.273285, 2006. DOI: 10.1051/animres:2006019.

MELE, M.; CONTARINI, G.; CERCACI, L.; SERRA, A.; BUCCIONI,A.; POVOLO, M.; CONTE, G.; FUNARO,A.;BANNI, S.; LERCKER, G.; SECCHIARI, P. Enrichment of Pecorino cheese with conjugated linoleic acid by feeding dairy ewes with extruded linseed: effect on fatty acid and triglycerides composition and on oxidative stability. International Dairy Journal, v.21, p.365-372, 2011. DOI: 10.1016/j.idairyj.2010.12.015.

MELE, M.; SERRA, A.; BUCCIONI, A.; CONTE, G.; POLLICARDO, A.; SECCHIARI, P. Effect of soybean oil supplementation on milk fatty acid composition from Saanen goats fed diets with different forage:concentrate ratios. Italian
Journal of Animal Science, v.7, p.297-312, 2008. DOI: 10.4081/ ijas.2008.297.

MIR, Z.; GOONEWARDENE, L.A.; OKINE, E.; JAEGAR, S.; SCHEER, H.D. Effect of feeding canola oil on constituents, conjugated linoleic acid (CLA) and long chain fatty acids in goats milk. Small Ruminant Research, v.33, p.137-143, 1999. DOI: 10.1016/S0921-4488(99)00016-4.

MORAND-FEHR, P.; FEDELE, V.; DECANDIA, M.; LE FRILEUX, Y. Influence of farming and feeding systems on composition and quality of goat and sheep milk. Small Ruminant Research, v.68, p.20-34, 2007. DOI: 10.1016/j. smallrumres.2006.09.019.

MUGHETTI, L.; SINESIO, F.; ACUTI, G.; ANTONINI, C.; MONETA, E.; PEPARAIO, M.; TRABALZA-MARINUCCI, M. Integration of extruded linseed into dairy sheep diets: effects on milk composition and quality and sensorial properties of Pecorino cheese. Animal Feed Science and Technology, v.178, p.27-39, 2012. DOI: 10.1016/j.anifeedsci.2012.09.005.

NUDDA, A.; BATTACONE, G.; BOAVENTURA NETO, O.; CANNAS, A.; FRANCESCONI, A.H.D.; ATZORI, A.S.; PULINA G. Feeding strategies to design the fatty acid profile of sheep milk and cheese. Revista Brasileira de Zootecnia, v.43, p.445-456, 2014. DOI: $10.1590 / \mathrm{S} 1516-35982014000800008$.

NUDDA, A.; BATTACONE, G.; USAI, M.G.; FANCELLU, S.; PULINA, G. Supplementation with extruded linseed cake affects concentrations of conjugated linoleic acid and vaccenic acid in goat milk. Journal of Dairy Science, v.89, p.277-282, 2006. DOI: 10.3168/jds.S0022-0302(06)72092-6.

NUTRIENT requirements of dairy cattle. $7^{\text {th }}$ ed. Washington: National Academic Press, 2001. 381p.

OLLIER, S.; LEROUX, C.; DE LA FOYE, A.; BERNARD, L.; ROUEL, J.; CHILLIARD, Y. Whole intact rapeseeds or sunflower oil in high-forage or high-concentrate diets affects milk yield, milk composition, and mammary gene expression profile in goats. Journal of Dairy Science, v.92, p.5544-5560, 2009. DOI: 10.3168/ jds.2009-2022.

PALMQUIST, D.L.; MOSER, E.A. Dietary fat effects on blood insulin, glucose utilization, and milk protein content of lactating cows. Journal of Dairy Science, v.64, p.1664-1670, 1981. DOI: 10.3168/jds.S0022-0302(81)82744-0.

PIRISI, A.; LAURET, A.; DUBEUF, J.P. Basic and incentive payments for goat and sheep milk in relation to quality. Small Ruminant Research, v.68, p.167-178, 2007. DOI: 10.1016/j. smallrumres.2006.09.009.

POL, M.V.; CASALS, R.; ALBANELL, E.; SUCH, X. Effect of supplementation with whole flax seed on milk yield and cheese quality of Manchega ewes breed. In: JORNADAS SOBRE PRODUCCIÓN ANIMAL, 9., 2001, Zaragoza. [Anales]. Zaragoza: Asociación Interprofesional para el Desarrollo Agrario, 2001. p.655-657.

PRIETO, N.; BODAS, R.; LÓPEZ-CAMPOS, Ó.; ANDRÉS, S.; LÓPEZ, S.; GIRÁLDEZ, F.J. Effect of sunflower oil supplementation and milking frequency reduction on sheep milk production and composition. Journal of Animal Science, v.91, p.446-454, 2013. DOI: 10.2527/jas.2012-5187. 
RABIEE, A.R.; BREINHILD, K.; SCOTT, W.; GOLDER, H.M.; BLOCK, E.; LEAN, I.J. Effect of fat additions to diets of dairy cattle on milk production and components: a meta-analysis and meta-regression. Journal of Dairy Science, v.95, p.3225-3247, 2012. DOI: $10.3168 /$ jds.2011-4895.

SAMPELAYO, M.R.S.; CHILLIARD, Y.; SCHMIDELY, P.; BOZA, J. Influence of type of diet on the fat constituents of goat and sheep milk. Small Ruminant Research, v.68, p.42-63, 2007. DOI: 10.1016/j.smallrumres.2006.09.017.

SAUVANT, D.; SCHMIDELY, P.; DAUDIN, J.J.; ST-PIERRE, N.R. Meta-analyses of experimental data in animal nutrition. Animal, v.2, p.1203-1214, 2008. DOI: 10.1017/S1751731108002280.

SCHMIDELY, P.; MORAND-FEHR, P.; SAUVANT, D. Influence of extruded soybeans with or without bicarbonate on milk performance and fatty acid composition of goat milk. Journal of Dairy Science, v.88, p.757-765, 2005. DOI: $10.3168 /$ jds.S00220302(05)72739-9.

SCHMIDELY, P.; SAUVANT, D. Taux butyreux et composition de la matière grasse du lait chez les petits ruminants: effets de l'apport de matières grasses ou d'aliment concentré. INRA Productions Animales, v.14, p.337-354, 2001.

SHINGFIELD, K.J.; BONNET, M.; SCOLLAN, N.D. Recent developments in altering the fatty acid composition of ruminant-derived foods. Animal, v.7, p.132-162, 2013. DOI: 10.1017/S1751731112001681.

SILVA, M.M.C. da; RODRIGUES, M.T.; BRANCO, R.H.; RODRIGUES, C.A.F.; SARMENTO, J.L.R.; QUEIROZ, A.C. de; SILVA, S.P. da. Suplementação de lipídios em dietas para cabras em lactação: consumo e eficiência de utilização de nutrientes. Revista Brasileira de Zootecnia, v.36, p.257-267, 2007. DOI: 10.1590/S1516-35982007000100030.

TEH, T.H.; TRUNG, L.T.; JIA, Z.H.; GIPSON, T.A.; OGDEN, K.B.; SWEENEY, T.F. Varying amounts of rumen-inert fat for high producing goats in early lactation. Journal of Dairy Science, v.77, p.253-258, 1994. DOI: 10.3168/jds.S0022-0302(94)76948-4.

TITI, H.H.; HASAN, Y.L.; AL-ISMAIL, K.; ZAKARIA, H.; TABBAA, M.J.; ABDULLAH, A.Y.; OBEIDAT, B.S. Response of
Shami goats and kids to variable levels of soyabean or sunflower oils in diet. Journal of Animal and Feed Sciences, v.20, p.493508, 2011.

TORAL, P.G.; FRUTOS, P.; HERVÁS, G.; GÓMEZ-CORTÉS, P.; JUÁREZ, M.; DE LA FUENTE, M.A. Changes in milk fatty acid profile and animal performance in response to fish oil supplementation, alone or in combination with sunflower oil, in dairy ewes. Journal of Dairy Science, v.93, p.1604-1615, 2010a. DOI: $10.3168 /$ jds.2009-2530.

TORAL, P.G.; HERVÁS, G.; GÓMEZ-CORTÉS, P.; FRUTOS, P.; JUÁREZ, M.; DE LA FUENTE, M.A. Milk fatty acid profile and dairy sheep performance in response to diet supplementation with sunflower oil plus incremental levels of marine algae. Journal of Dairy Science, v.93, p.1655-1667, 2010b. DOI: 10.3168/jds.20092769.

WU, Z.; HUBER, J.T. Relationship between dietary fat supplementation and milk protein concentration in lactating cows: a review. Livestock Production Science, v.39, p.141-155, 1994. DOI: 10.1016/0301-6226(94)90180-5.

ZERVAS, G.; FEGEROS, K.; KOYTSOTOLIS, K.; GOULAS, C.; MANTZIOS, A. Soy hulls as a replacement for maize in lactating dairy ewe diets with or without dietary fat supplements. Animal Feed Science and Technology, v.76, p. 65-75, 1998. DOI: 10.1016/S0377-8401(98)00209-0.

ZHANG, R.H.; MUSTAFA, A.F.; ZHAO, X. Blood metabolites and fatty acid composition of milk and cheese from ewes fed oilseeds. Canadian Journal of Animal Science, v.86, p.547-556, 2006a. DOI: 10.4141/A06-038.

ZHANG, R.H.; MUSTAFA, A.F.; ZHAO, X. Effects of feeding oilseeds rich in linoleic and linolenic fatty acids to lactating ewes on cheese yield and on fatty acid composition of milk and cheese. Animal Feed Science and Technology, v.127, p.220-233, 2006b. DOI: 10.1016/j.anifeedsci.2005.09.001

ZHANG, R.H.; MUSTAFA, A.F.; ZHAO, X. Effects of flaxseed supplementation to lactating ewes on milk composition, cheese yield, and fatty acid composition of milk and cheese. Small Ruminant Research, v.63, p.233-241, 2006c. DOI: 10.1016/j. smallrumres.2005.02.024.

Recibido el 27 de julio de 2014 y aceptado el 14 de julio de 2015 